\title{
Effets cumulés sur les poissons migrateurs, état et restauration des grands axes de migration du bassin de la Loire
}

\author{
Cumulated effects on migratory fish, \\ status and restoration of the main migratory axes \\ in the Loire basin
}

\section{Pierre Steinbach}

Conseil Supérieur de la Pêche, Délégation Régionale Centre, Pays de la Loire, Poitou Charente - Cellule Opérationnelle Plan Loire, Service de Bassin Loire Bretagne, 5 avenue Buffon, BP 640745064 ORLEANS Cedex 2, France.

E-mail : csp@centre.environnement.gouv.fr

Résumé. - Les facteurs limitants des populations de poissons migrateurs du bassin de la Loire résultent de l'effet cumulé des obstacles qui se manifestent le long des axes de migration. Ces impacts sont exacerbés par la longueur des parcours qui séparent l'estuaire des zones amont les plus productives. Les effets de l'eutrophisation qui s'aggravent vers l'aval au fil des apports polluants, jusqu'au bouchon vaseux de l'estuaire, sont également conditionnés par la dimension du bassin. Dans ces conditions, les stocks ligériens restent fortement tributaires de l'hydrologie. Les variations inter-annuelles de débit peuvent réduire temporairement l'emprise des facteurs limitants ou, au contraire, conduire à des situations de crise menaçant la pérennité des espèces, comme lors de la période de sécheresse qui a marqué le début des années 1990. Compte tenu du poids des effets cumulés, la restauration de ces populations fragiles nécessite la mise en œuvre d'opérations fortes en matière de transparence migratoire, telle que l'effacement de barrages et la réalisation de passes à grand débit conçues en s'inspirant de l'hétérogénéité des faciès naturels.

Mots clés. - Effets cumulés, poissons migrateurs, Loire.

Abstract. - The population-limiting factors on migratory fish in the Loire Basin are the result of the cumulated effects of obstacles encountered all along migratory axes. These impacts are reinforced by the distance between the estuary from the most productive upstream areas. The effects of eutrophication that become aggravated downstream from pollutants, up to the maximum of turbidity in the estuary, are also determined by the size of the system. In these conditions, Loire stocks remain highly dependent on hydrology. Year to year variations of flow-rate can temporarily reduce the impact of human factors, or on the contrary lead to crisis situations that menace the future of species. A good 
example of this is the drought that marked the early 1990s. In light of the importance of cumulated effects, the restoration of these fragile populations requires massive efforts that remain transparent to migration, such as the removal of dams and conceiving high flow-rate channels to mimic the natural habitats.

Key words. - Cumulated effects, migratory fish, Loire.

\section{INTRODUCTION}

Le bassin de la Loire couvre $120000 \mathrm{~km}^{2}$, soit un cinquième du territoire français. Parfaitement centré sur la façade occidentale de l'Europe, il bénéficie d'une situation biogéographique privilégiée par rapport à l'aire de répartition des espèces amphihalines de l'Atlantique nord.

Ce système fluvial est fréquenté régulièrement par six grands migrateurs amphihalins. Parmi les espèces présentes au début du siècle, seul l'esturgeon (Acipenser sturio) a totalement disparu depuis la fin des années 1940. L'espèce la plus emblématique du bassin est le saumon atlantique (Salmo salar), dont la population de l'axe Loire-Allier correspond à la dernière souche d'Europe occidentale inféodée aux grands systèmes fluviaux. Ce stock relictuel est essentiellement constitué de grands saumons avec $43 \%$ de géniteurs âgés de 3 ans de mer, $55 \%$ âgés de 2 ans de mer et seulement de $2 \%$ de castillons (BACH et al., 2001). La truite de mer (Salmo trutta trutta) est également présente mais sa population est nettement moins bien caractérisée. L'anguille (Anguilla anguilla) est très largement répartie à l'intérieur du bassin. Pour les aloses, la plus grande partie du stock ligérien est constituée par la grande alose (Alosa alosa). L'alose feinte (Alosa fallax) ne représente que $1 \%$ à $5 \%$ des populations du bassin (MENESSONBOISNEAU, 1990) et se cantonne à la partie aval du réseau hydrographique. En ce qui concerne les lamproies, l'essentiel du stock est constitué par la lamproie marine $(\mathrm{Pe}$ tromizon marinus). Les signalements de lamproies fluviatiles (Lampetra fluviatilis) dans le secteur aval ne permettent pas de confirmer l'existence d'une population correspondant à cette dernière espèce dans le bassin de la Loire (STEINBACH, 1997).

\section{CAPACITÉ D'ACCUEIL ET POTENTIEL PRODUCTIF}

L'aire de répartition de l'anguille dans le bassin de la Loire témoigne de la grande capacité d'accueil de ce réseau hydrographique. La quasi totalité des $100000 \mathrm{~km}$ du réseau est potentiellement exploitable pour le développement de cette espèce. L'essentiel de sa zone de répartition est constituée de cours d'eau de plaine et de basse montagne, au-dessous de 1000 m d'altitude. Ainsi, l'ensemble du bassin est potentiellement colonisable à plus de $800 \mathrm{~km}$ de la mer, au-delà du barrage de Poutès 
Monistrol (axe Loire-Allier) où l'on observe encore des migrations d'anguilles, dans le dispositif de franchissement mis en place pour le saumon (ascenseur).

La capacité d'accueil de la Loire et de ses affluents repose sur la densité de drainage de son réseau hydrographique, particulièrement forte en amont du bec d'Allier: 0,99 $\mathrm{km}$ de cours d'eau $/ \mathrm{km}^{2}$ (SOUCHON et al., 2000), ainsi que sur de vastes zones humides. Ces dernières sont très développées à l'aval, dans la région des Pays de la Loire (Grande Brière, Grand Lieu, marais Breton...), mais aussi à l'intérieur du bassin, dans les dépressions elluviales de la région Centre (Brenne et Sologne en particulier).

On observe cependant d'importants défauts de répartition des espèces migratrices sur les versants du massif central. Ces vides résultent de l'impact des grands aménagements hydroélectriques du $20^{\text {ème }}$ siècle qui ferment la plupart des gorges donnant accès au réseau centralien :

- complexe de l'lle Jourdain sur la Vienne

- complexe d'Eguzon sur la Creuse

- Prat / Rochebut sur le Cher

- Villerest / Grangent sur la Loire amont.

Au plan qualitatif, pour les espèces exigeantes en matière d'habitat de reproduction, les secteurs qui offrent le meilleur potentiel productif se situent sur les versants du massif central. Ainsi, sur les deux axes qui restent libres d'obstacle rédhibitoire
(Gartempe, Allier et Arroux), les parcours de migration du saumon s'étendent sur au moins $350 \mathrm{~km}$ pour ce qui concerne les frayères les plus proches de la mer sur la Gartempe poitevine et $950 \mathrm{~km}$ pour les zones de reproduction les plus éloignées sur l'Allier lozérien (Figure 1).

La grande alose et la lamproie marine sont capables d'exploiter des zones de reproduction moins apicales. Pour ces espèces, les frayères naturelles les plus accessibles se situent tout de même à plus de $250 \mathrm{~km}$ de l'océan et la distribution des habitats les plus intéressants s'étend jusque dans les hauts bassins, recouvrant en partie l'aire de reproduction du saumon (jusque dans les gorges de l'Allier).

La dominante sableuse du lit de la Loire moyenne ne permet pas la formation de radiers naturels caillouteux intéressants pour la reproduction des espèces potamotoques. Par conséquent, au-delà des confluences de la Vienne et du Cher, les géniteurs de grande alose et de lamproie doivent dépasser le bec d'Allier pour trouver des zones de frayères favorables.

\section{IMPACTS DES EFFETS CUMULÉS}

La longueur des axes de migration entre la mer et les zones amont les plus productives constitue la principale caractéristique du réseau ligérien. C'est de là que découle la valeur patrimoniale des stocks ligériens à l'instar du saumon de l'axe Loire- 


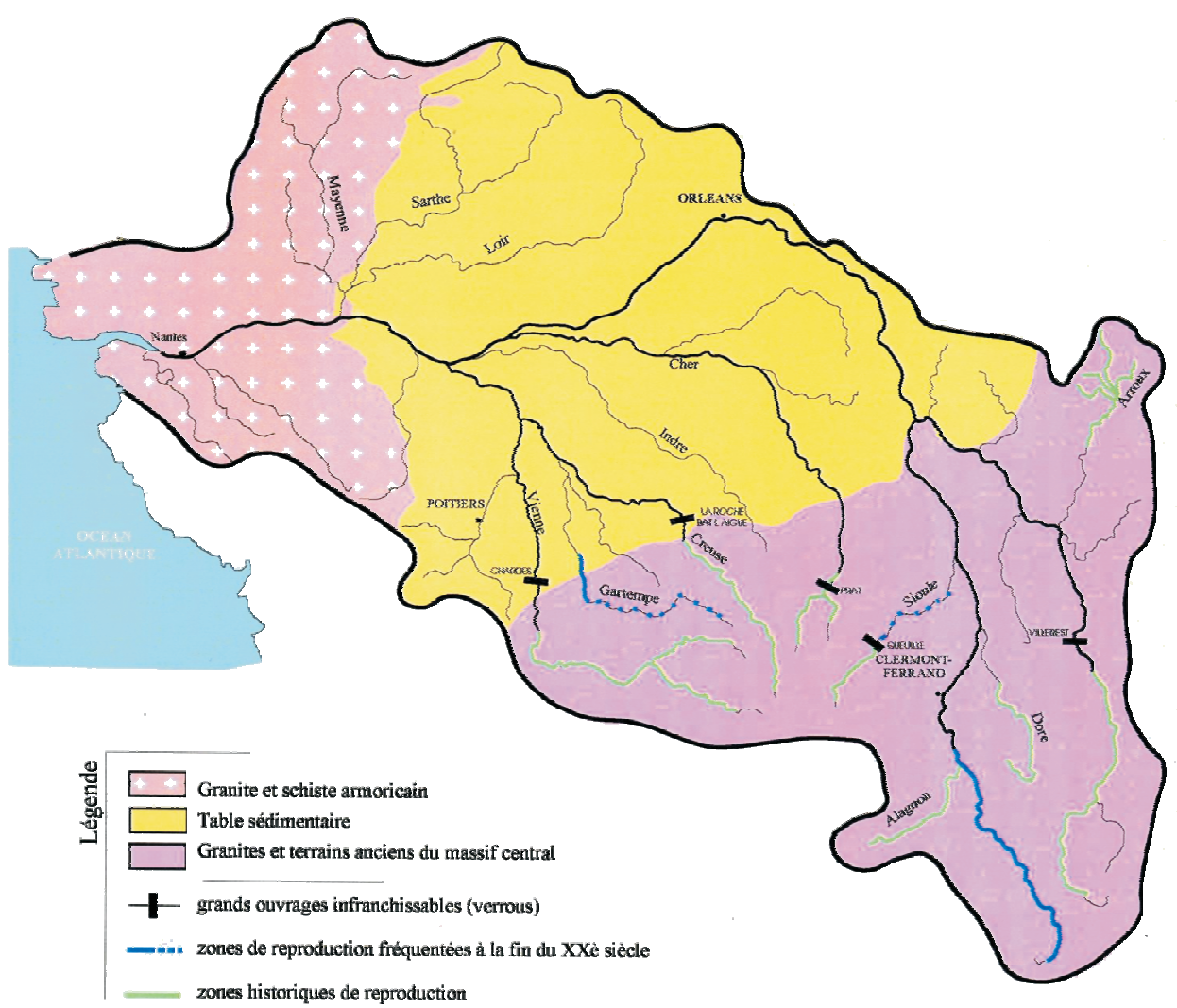

Fig. 1. - Répartition des zones de reproduction du saumon dans le bassin de la Loire.

Fig. 1. - Distribution of salmon spawning areas in the Loire basin.

Allier qui constitue, pour l'Europe, la dernière souche endémique de grands saumons atlantiques. C'est aussi de cette dimension fluviale que résultent les principales difficultés de conservation et de restauration des populations amphihalines du bassin, du fait de la démultiplication des contraintes et des impacts qui se succèdent le long des circuits de migration.

\subsection{Conditions de migration anadrome - Cas de l'alose et de la lamproie}

Même pour la grande alose et la lamproie marine, capables d'exploiter des zones de frayères potentielles sur le cours médian des circuits de migration, le nombre des obstacles qui jalonnent les axes du bassin peut 
compromettre sérieusement l'accès aux frayères, à la fois en terme d'effectif migrant et en terme de retard migratoire. Suivant son taux de franchissabilité, chaque obstacle réduit la fraction de population qui peut poursuivre sa migration. Même si la proportion de poissons bloqués est faible ponctuellement, la réduction exponentielle des effectifs en fonction du nombre d'obstacles à franchir peut conduire à un abattement drastique des effectifs en fin de parcours (LARINIER et al., 1994). En outre, compte tenu des délais imposés par la biologie des espèces, l'accumulation des retards migratoires et des atteintes physiologiques concédés à chaque franchissement affaiblit la fraction migrante et réduit ses chances d'accès aux zones de reproduction.

Sur les affluents de la Maine (Mayenne, Sarthe, Loir), qui constituent les axes les plus proches de la mer, les géniteurs ont une dizaine d'obstacles à franchir pour atteindre les premières frayères et une cinquantaine pour couvrir l'ensemble de l'aire de reproduction disponible.

Sur le faisceau centralien des affluents convergents en aval de Tours (bassins Vienne/Cher), les aires de reproduction sont limitées par les grands verrous hydroélectriques. Selon les circuits de migration qui restent disponibles, les cumuls d'obstacles atteignent 10 à 40 ouvrages au niveau des limites amont des zones potentielles de reproduction. Cependant, depuis l'effacement du barrage de Maisons Rouges, le bassin de la Vienne offrent des frayères directement accessibles, sans aucun obstacle depuis l'estuaire jusqu'aux barrages de Châtellerault sur la branche Vienne et Descartes sur la branche Creuse (Figure 2). Cette situation constitue un cas de figure exceptionnel dans le contexte ligérien.

Sur le bassin amont, les géniteurs doivent parcourir plus de $500 \mathrm{~km}$ pour atteindre les premiers sites de fraie, après un minimum de 7 obstacles à franchir en Loire moyenne et un maximum d'une vingtaine d'obstacles pour couvrir l'ensemble des zones de reproduction disponibles.

\subsection{Conditions de migration catadrome}

Le résultat des effets cumulés peut être aussi pénalisant dans le sens de la dévalaison.

A titre d'exemple, l'impact des micro-centrales hydroélectriques équipant les barrages de la Mayenne augmente les facteurs de mortalité sur la fraction de population d'anguilles qui dévale l'axe principal pour aller se reproduire en mer, après avoir colonisé le bassin durant sa phase de croissance. Les taux d'entraînement et de mortalité des adultes passant par les turbines n'ont pas été étudiés lors de la mise en place des installations. Néanmoins, le phénomène peut prendre des proportions importantes par effet cumulatif. Dans l'hypothèse où $80 \%$ des anguilles échapperaient à l'impact des turbines sur ce type d'ouvrage, in fine le taux 


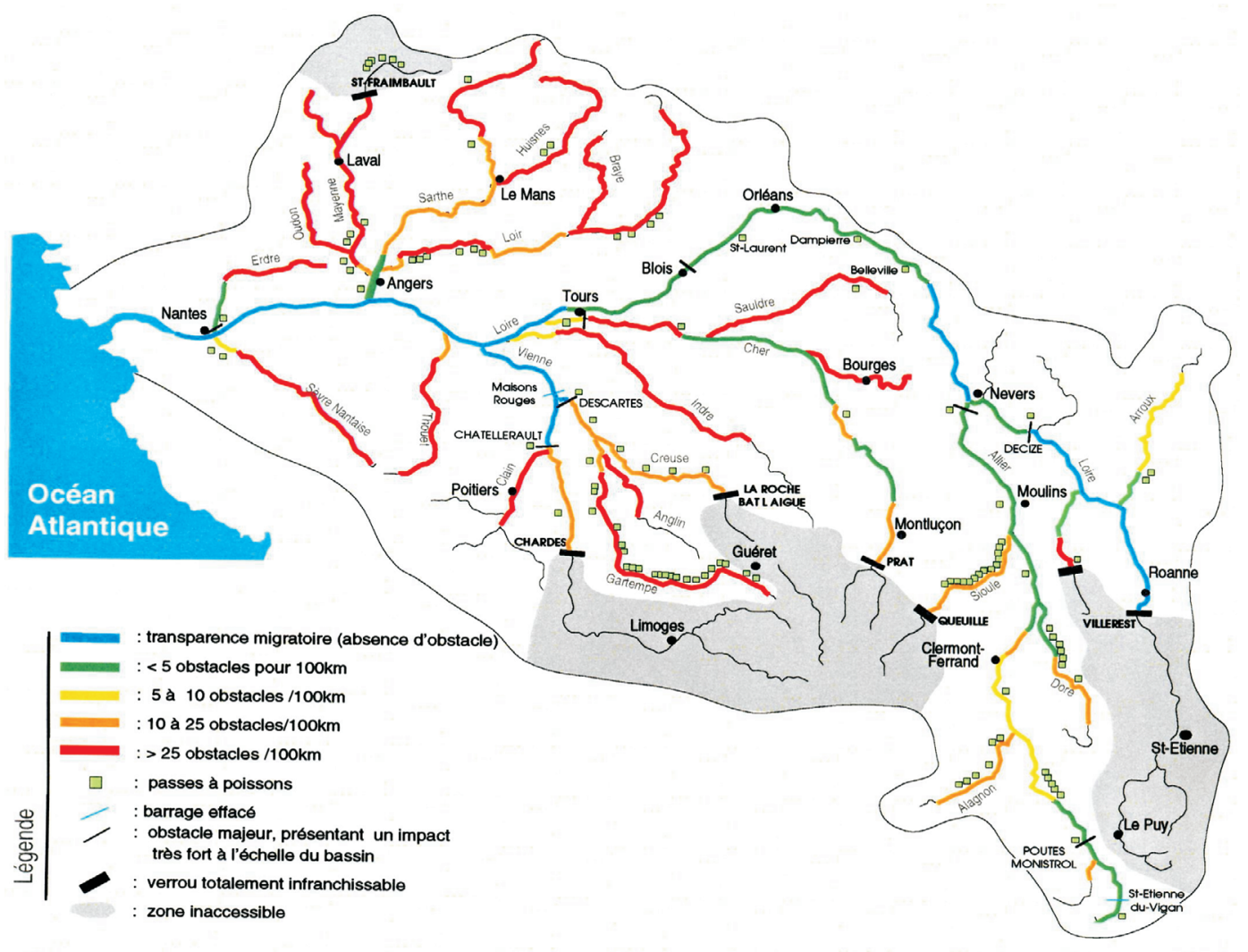

Fig. 2. - Densité d'obstacle et état des principaux axes de migration du bassin de la Loire.

Fig. 2. - Obstacle density and state of main migratory axes in the Loire basin.

de survie théorique serait inférieur à $1 \%$ après le franchissement des 21 micro-centrales qui jalonnent l'axe Mayenne. L'impact des ouvrages hydroélectriques sur les anguilles d'avalaison doit cependant être relativisé en fonction des densités de populations présentes en tête de bassin, celles-ci étant elles-mêmes limitées par l'effet des barrages qui ré- duisent les possibilités de colonisation au stade de la montaison.

La problématique des effets cumulés sur les migrations catadromes n'a pas encore fait l'objet d'évaluation précise dans le bassin de la Loire. II serait intéressant de mener des travaux de modélisation à ce sujet, comme cela a pu être étudié dans d'autres bassins (exemple : dévalai- 
son des smolts sur les rivières à saumons du bassin de la Garonne).

Outre les mortalités directes occasionnées par les équipements hydroélectriques, les retenues des ouvrages aménagés sur les axes de migration se traduisent par des ruptures morphodynamiques qui perturbent et ralentissent la progression des poissons dont la dévalaison est conditionnée par le courant. Les impacts indirects qui en résultent par prédation ou par retard migratoire à l'intérieur des retenues s'accumulent au même titre que les effets observés à la montaison. Le phénomène n'est pas négligeable dans le bassin de la Loire compte tenu de la longueur de ses circuits de migration et la situation de son estuaire. En effet, certains stades dévalants doivent atteindre rapidement le milieu marin avant la formation du « bouchon vaseux » à la fin du printemps (cas du saumon en particulier).

\subsection{Qualité de l'eau}

A l'instar des contraintes de libre circulation, les problèmes rencontrés en matière de qualité d'eau s'amplifient en fonction de la dimension du réseau, au fil des apports polluants qui s'accumulent depuis les têtes de bassin jusqu'à l'estuaire.

La qualité générale des eaux suit globalement le profil longitudinal du bassin. Elle est satisfaisante sur les versants du massif central excepté localement en aval d'agglomérations comme Montluçon sur le Cher ou
Saint-Etienne sur la Loire, ainsi qu'au niveau des grandes retenues de Grangent ou de Villerest (ANONYME, 2001). Sur les parties moyennes du bassin, le cumul des pollutions dégrade progressivement la qualité des eaux: rejets des agglomérations comme Orléans et Tours, pollutions diffuses de l'agriculture céréalière des tables calcaires du Centre, de la polyculture et des élevages intensifs du Massif armoricain.

La forme de pollution la plus notable résulte de la surcharge des cours d'eau en éléments fertilisants. Celle-ci se manifeste par une tendance très marquée à l'eutrophisation, aboutissant à une situation particulièrement critique au niveau de l'estuaire de la Loire. Cet exutoire connaît tous les ans des crises d'anoxie qui forment un écran physico-chimique infranchissable en période estivale. En année hydrologique normale, l'obstacle du bouchon vaseux s'installe en aval de Nantes du mois de juin au mois d'octobre. Cette saison critique coïncide avec la période biologique la moins active pour ce qui concerne le déplacement des espèces amphihalines en estuaire. Cependant, les processus d'eutrophisation et de désoxygénation varient largement en fonction de l'hydraulicité. L'emprise de l'altération est exacerbée en situation hydrologique défavorable. La formation de l'écran physico-chimique déborde alors sur les périodes sensibles de migration (printemps, automne). A l'inverse, le bouchon vaseux s'estompe en cas de forte hydraulicité. Ces effets cumulés 
qui se manifestent au niveau de l'exutoire du bassin constituent vraisemblablement un des premiers facteurs limitants des populations amphihalines de la Loire. Toutefois, l'impact spécifique de cette altération n'a pas encore été étudié sur cet estuaire, hormis en ce qui concerne la migration des mulets (SAURIAU, 1990).

\section{SYNTHÈSE DES CONTRAINTES PHYSIQUES ET DES EFFETS CUMULÉS}

La situation des axes du bassin et leur potentiel migratoire peuvent se résumer à travers l'expression de deux principaux facteurs limitants (Figure 3) :

- la distance à la mer pour ce qui concerne les contraintes naturelles,

- l'accumulation des retenues de barrage pour ce qui concerne la somme des impacts affectant l'intégrité de l'hydrosystème et la dégradation des habitats utilisés par les poissons migrateurs.

Le niveau d'altération du milieu s'exprime simplement par le taux d'étagement artificiel des cours d'eau. Ce métrique permet de distinguer :

- la part de dénivelé naturel annihilée par le remous des barrages

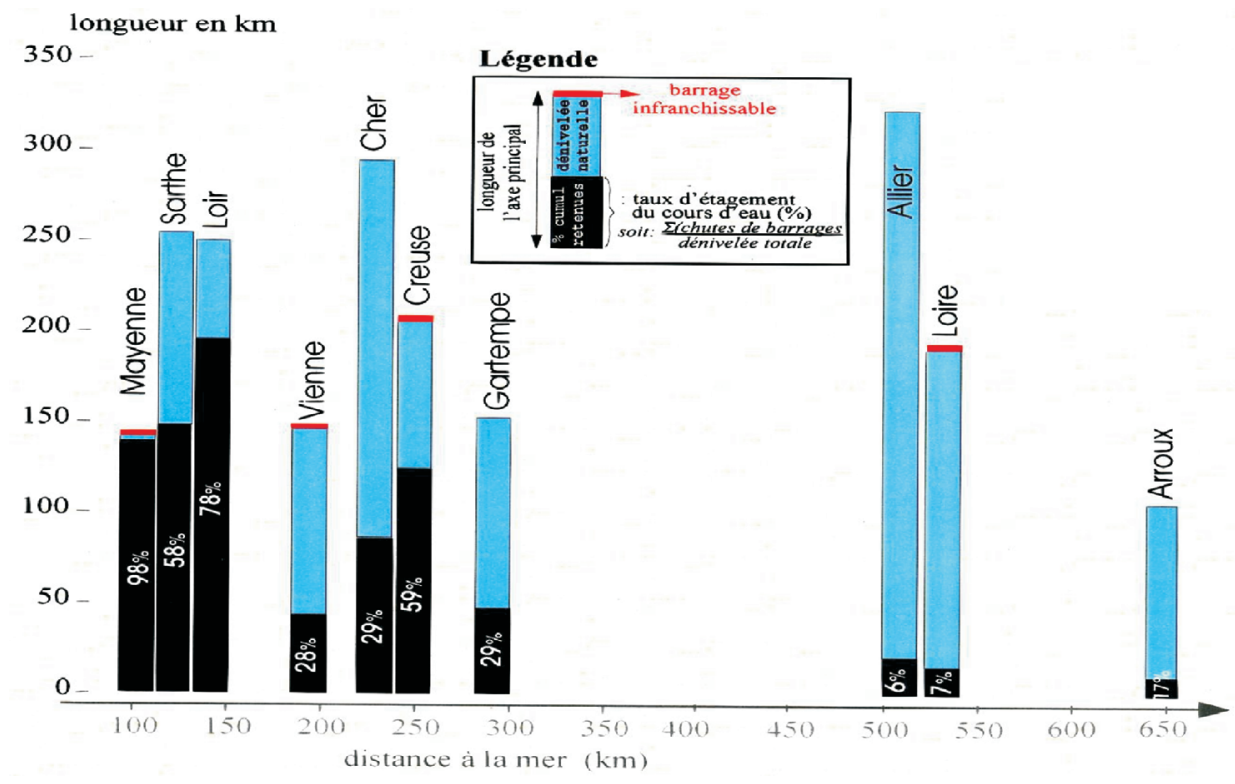

Fig. 3. - Potentialités des axes migratoires pour les poissons migrateurs.

Fig. 3. - Potential of the main migratory course. 
(pourcentage correspondant au cumul des hauteurs de chute),

- la part de dénivelé naturel non touchée, c'est-à-dire le potentiel d'écoulement libre qui reste disponible pour la formation des frayères (radiers naturels) et totalement transparente pour le déroulement des migrations.

Cette simple représentation par addition des chutes intègre la plupart des altérations et leurs effets cumulés:

- les impacts à la montaison liés à la hauteur et au nombre des barrages à franchir (blocages et retards migratoires),

- les impacts à la dévalaison et la perte des frayères potentielles liés à l'emprise des retenues (mortalités induites, retards migratoires et disparition des faciès productifs par ralentissement des écoulements et colmatage des fonds).

\section{ILLUSTRATION DES EFFETS CUMULÉS SUR L'ÉTAT DES STOCKS D'ALOSES}

Le stock de grandes aloses du bassin de la Loire suit une dynamique de population particulière. Entre 1984 et 1998 , le suivi des captures réalisées en Loire moyenne par un réseau de pêcheurs échantillonneurs au filet barrage a montré qu'il n'existe pas de relation stock-recrutement classique pour les aloses du système fluvial ligérien. Selon l'indice d'abondance mis au point à partir de cette pê- cherie, l'importance des cohortes est fortement dépendante de l'hydraulicité printanière de leur année de naissance (MENESSON - BOISNEAU et al., 1999).

On observe le même phénomène sur l'indice d'abondance correspondant aux captures totales déclarées par les pêcheurs professionnels fluviaux de l'estuaire (Figure 4). Les résultats de cette pêcherie permettent d'apprécier la variabilité inter-annuelle du stock ligérien dans la mesure où l'on dispose d'une série chronologique suffisamment longue (22 ans) avec une pêcherie suffisamment stable en terme d'effectif (50 pêcheurs) et de métier de pêche (filet dérivant).

Les captures totales présentent des fluctuations inter-annuelles d'un facteur dix, passant de 0,8 tonnes entre 1984 et 1986 à 6-7 tonnes entre 1987 et 1989. Avec un décalage correspondant au pas de temps d'une génération ( 5 ans en moyenne), ces variations de production confirment la relation entre le recrutement et les années de forte hydraulicité :

- quel que soit le niveau du stock, le recrutement est fort (1988) une génération après l'avènement d'un printemps exceptionnellement humide (1983), en réponse aux hautes eaux qui réduisent les effets cumulés et permettent l'accès aux frayères plus productives du cours amont des grands axes ;

- en revanche, le recrutement est faible (1995-1998) après une période d'hydraulicité défavorable (printemps secs 1990-1993) même 


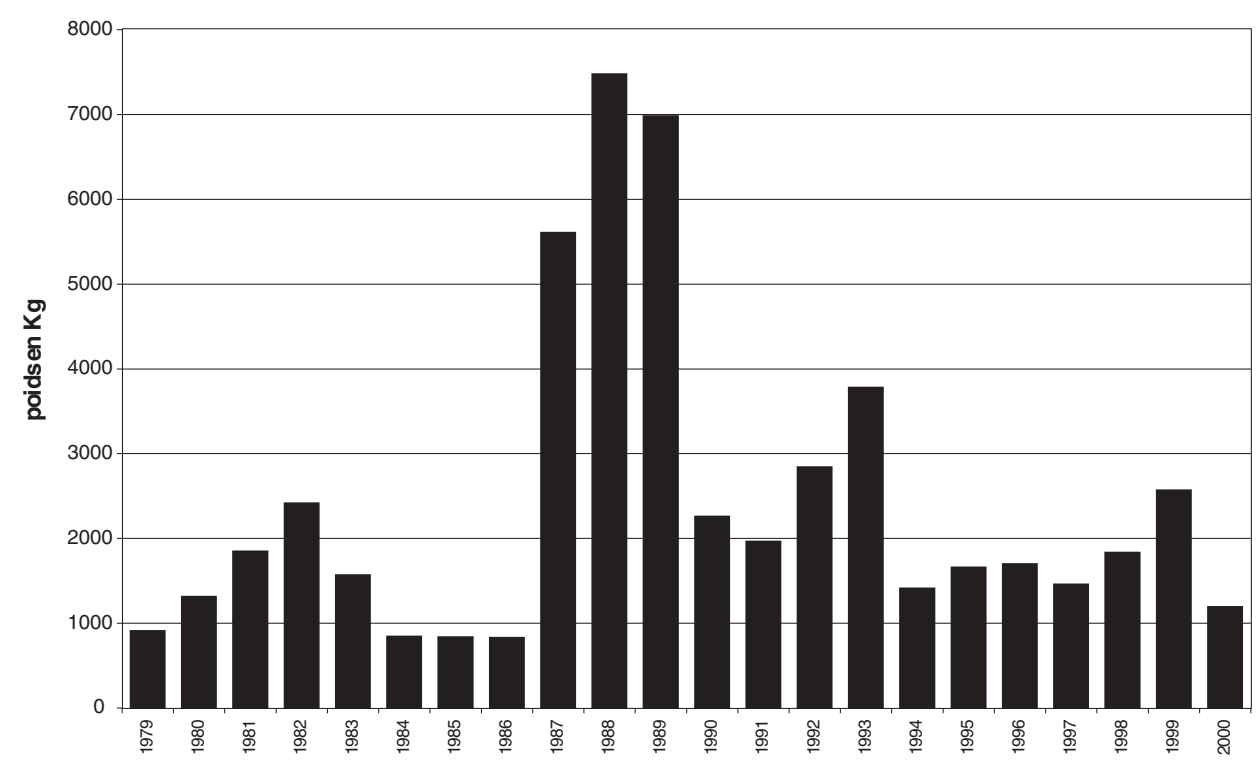

Fig. 4. - Poids total des captures d'aloses déclarées par les pêcheurs professionnels fluviaux de Loire-Atlantique.

Fig. 4. - Total weight of shad catches declared by fresh water professional fishermen of Loire atlantique.

si le nombre de géniteurs était important en estuaire.

L'état des stocks est suivi de façon plus précise par comptage des géniteurs au niveau des stations de contrôle installées sur les passes à poissons, plus haut sur les axes de migration. Les variations inter-annuelles observées à ce niveau du réseau hydrographique sont encore plus importantes. Comptabilisé à Vichy (Figure 5), le nombre de géniteurs accédant aux frayères du bassin amont ne varie pas d'un facteur 10 comme en estuaire. A ce niveau du réseau, les variations interannuelles peuvent atteindre un facteur 100 (1997/1998) en réponse aux effets cumulés qui interviennent, non seulement en fonction de l'hydraulicité de l'année de naissance des cohortes, mais aussi en fonction des débits rencontrés par les recrues au moment de migration vers les frayères amont.

\section{6 ÉVOLUTION DES EFFETS CUMULÉS SUR L'AXE PRINCIPAL}

Jusqu'à la fin des années 60 , la Loire bénéficiait d'une transparence migratoire totale. Le premier obstacle rencontré par les poissons migrateurs sur l'axe principal était le barrage de Decize situé en Bourgogne, à $600 \mathrm{~km}$ de la mer. Les conditions de libre circulation étaient encore relati- 


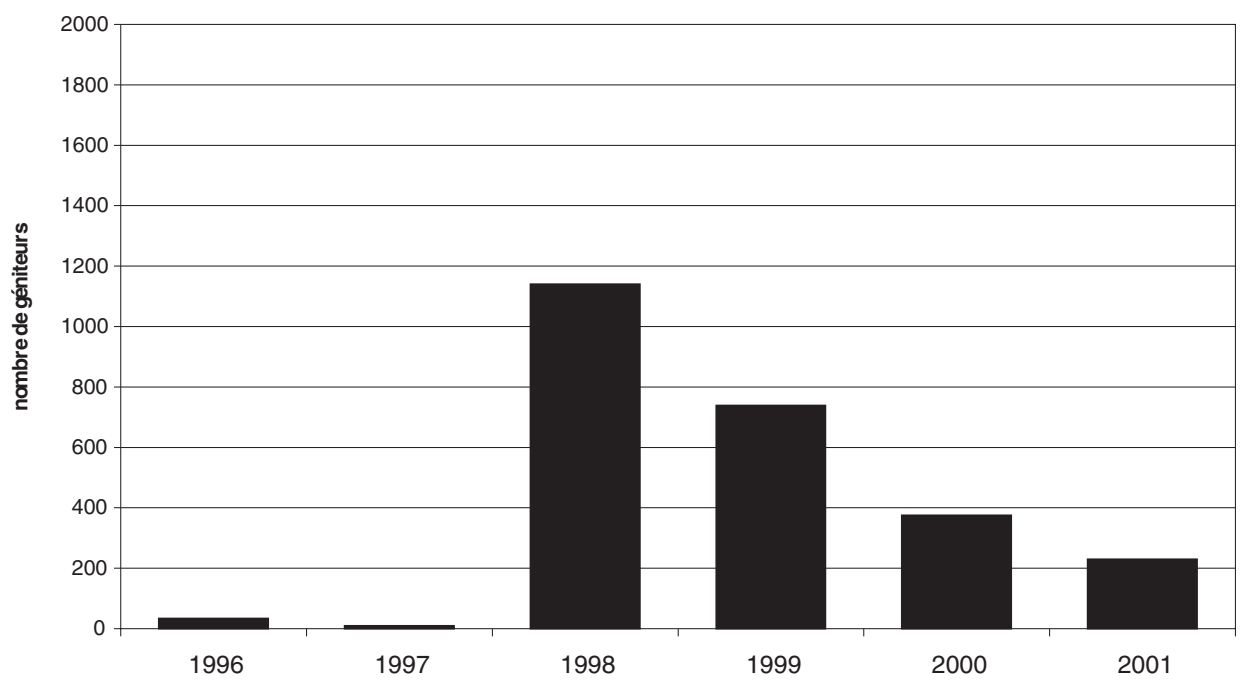

Fig. 5. - Nombre d'aloses comptabilisées à Vichy sur l'axe Allier depuis la mise en service de la station de contrôle des migrations anadromes.

Fig. 5. - Number of shads counted at Vichy on Allier river since the anadromus migration monitoring device was installed.

vement bien préservées sur l'axe alors que débutait, au milieu des années 70 , le premier plan national en faveur des poissons migrateurs. C'est pourtant à partir de la période de lancement du «plan saumon» que la fluidité migratoire de la Loire moyenne s'est dégradée progressivement, sous l'effet conjugué des aménagements et de l'enfoncement du lit du fleuve (extraction de granulats). Outre les radiers des anciens ponts affouillés, les obstacles les plus difficilement franchissables pour les poissons qui se reproduisent en amont du bec d'Allier correspondent aux trois seuils de prise d'eau construits pour l'alimentation des centrales nucléaires (Saint-Laurent-desEaux, Dampierre-en-Burly, Bellevillesur-Loire).
L'altération de la transparence migratoire de la Loire moyenne n'est probablement pas étrangère au déclin des stocks ligériens observé à la fin du $20^{\text {ème }}$ siècle. Aussi, la réalisation des objectifs de restauration des populations d'aloses, de saumons, de lamproies et d'anguilles passe par le rétablissement de la libre circulation sur l'ensemble de l'axe, conformément aux obligations de résultat qui s'imposent à tous les maîtres d'ouvrages depuis 1986, en vertu de l'article L. 432-6 du code de l'Environnement.

En fait, le rétablissement de fluidité migratoire n'a véritablement débuté qu'en 1998 avec les travaux entrepris dans le cadre du Plan Loire (radier du Pont Wilson à Tours) et surtout dans le cadre de la mise en conformité des 
ouvrages EDF, à l'occasion des renouvellements d'autorisation temporaire d'occupation du domaine public fluvial. Après l'aménagement des passes à poissons de Dampierre (1998) et Saint-Laurent-des-Eaux (2000), cet effort de traitement des obstacles de la Loire moyenne devrait aboutir prochainement, avec le réaménagement du dispositif de franchissement de Belleville sur la Loire et l'échéance de l'autorisation administrative du barrage mobile de Blois.

\section{CAS DU SAUMON}

Les difficultés rencontrées par les poissons migrateurs amphihalins le long de leurs circuits de migration en eau douce illustrent bien la notion d'effets cumulés. En estuaire et en milieu marin, les impacts cumulatifs sont plus difficiles à cerner mais ces derniers ont probablement une incidence aussi forte sur l'état de certains stocks.

La dernière population de saumons atlantiques caractéristique des grands systèmes fluviaux européens à failli disparaître sur l'axe Loire-Allier lors de la sécheresse qui a marqué le début des année 1990. C'est en 1994 que la pêche a été totalement interdite dans ce bassin et qu'un plan de sauvegarde a été lancé dans le cadre du Plan Loire Grandeur Nature.

Depuis le réaménagement du dispositif de franchissement du pontbarrage de Vichy et la mise en service de la station de contrôle associée à cette ouvrage, on observe que les conditions hydrologiques et leur effets modulateurs sur les impacts en milieu continental (obstacles physiques et physico-chimiques, captures accidentelles...) se traduisent surtout par des variations au niveau de la répartition des géniteurs le long de l'axe. Par rapport au nombre de saumons comptabilisés à Vichy, la proportion de reproducteurs qui accèdent aux frayères du haut Allier en amont de Poutès-Monistrol varie de $10 \%$ à $30 \%$ selon l'hydraulicité du cours d'eau (ANONYME, 2002).

Pour autant, en terme d'effectif total, les résultats observés ne présentent pas de relation significative avec l'hydrologie. Malgré l'augmentation des déversements de juvéniles et la variabilité des conditions de migration en milieu continental, les remontées de géniteurs apparaissent stables à hauteur de Vichy. Le nombre d'adultes comptabilisés se situe autour de 400 par an et par cohorte, ce qui représente un quart du seuil de conservation correspondant à la capacité d'accueil des zones de reproduction situées en amont (ANONYME, 2002; MINSTER et BOMASSI, 1999).

Le suivi et la gestion de cette population nécessitent une meilleure connaissance des taux de survie en mer. Cette phase marine du cycle vital est d'autant plus importante chez le saumon du bassin de la Loire que sa durée de séjour en mer est exceptionnellement longue (2 à 3 ans) et que ses zones d'engraissement sont très éloignées de l'estuaire (4000 à $5000 \mathrm{~km}$ ). Cette situation augmente 
sensiblement le champ d'action des impacts cumulatifs déjà très prégnants en milieu continental.

Compte tenu des facteurs de mortalité qui se multiplient sur l'ensemble de son parcours biologique, cette population menacée est soutenue par reproduction artificielle pour reconstituer un niveau de stock correspondant à la capacité d'accueil et au potentiel productif naturel du bassin. Dans le cadre de la première étape du Plan Loire, l'effort d'alevinage a été multiplié par trois pour atteindre 93000 Equivalents Saumoneaux Sauvages (CARMIE, 1997) en moyenne annuelle, entre 1995 et 2001 . Avec le fonctionnement de la nouvelle salmoniculture construite à Chanteuges (43), cet effort de soutien d'effectif devrait approcher 150000 Equivalents Saumoneaux Sauvages, majoritairement déversés sous forme de smolts.

\section{RÉTABLISSEMENT DE LA TRANSPARENCE MIGRATOIRE}

Depuis le premier plan de restauration lancé en 1976, plus de 100 dispositifs de franchissement ont été aménagés sur les principaux circuits de migration du bassin (Figure 2). Cependant, compte tenu de la multiplication des obstacles et des limites d'efficacité des dispositifs de franchissement, notamment en terme de conception et de gestion des ouvrages, il a fallu attendre les actions fortes mises en œuvre à la fin des années 1990 pour observer des ré- sultats significatifs en matière de reconquête migratoire.

La solution systémique la plus efficace est celle qui a été appliquée en 1998 dans le cadre du Plan Loire, par effacement des barrages de SaintEtienne-du-Vigan et Maisons Rouges.

La suppression des barrages permet de rétablir des conditions de transparence migratoire totale. Dans le même temps, elle permet de recréer des habitats naturels de reproduction. Dès l'année suivant les travaux d'effacement, des frayères à saumons ont été réactivées dans l'ancienne retenue du barrage de Saint-Etienne-du-Vigan. De même, les radiers naturels noyés sous l'emprise de l'ancienne retenue de Maisons Rouges ont immédiatement été réinvestis par la lamproie marine, avec une forte densité de nids de ponte observée sur ces faciès revitalisés (CHAPON, 1999).

En dépit de la "rugosité migratoire » du réseau amont, la levée complète de ce verrou qui marquait l'accès au bassin de la Vienne a également permis la reconquête d'une grande partie de l'aire de répartition de l'espèce. Aidée par une hydrologie favorable, l'effet de réouverture a été suffisamment fort pour que le front de migration de la lamproie marine remonte jusqu'au pied du complexe hydroélectrique d'Eguzon (barrage de la Roche-Bât-L'Aigue). En revanche, les résultats de reconquête migratoire sont moins significatifs pour ce qui concerne la grande alose qui ne 
bénéficie pas des mêmes aptitudes au franchissement des obstacles.

C'est pour répondre aux exigences de cette espèce limitée par ses capacités de nage, son comportement rétif face aux écoulements turbulents, son incapacité au saut et ses faibles aptitudes au repérage des voies de franchissement, qu'un nouveau concept de passe à poissons a été mis au point pour équiper les seuils fixes de la Loire moyenne. Celui-ci consiste à réaliser des dispositifs attractifs au moyen de passes à grands débits structurées par des seuils rugueux à double pendage.

\section{CONCLUSION}

Le bassin de la Loire présente une extension géographique importante avec une forte diversité hydro-écologique. Cette richesse suit le déterminisme de la géologie, du relief et du gradient qui éloigne les hauts bassins continentaux de la façade océanique. $\mathrm{Ce}$ réseau hydrographique développe des capacités d'accueil et de production particulièrement vastes et diversifiées pour les populations de poissons migrateurs amphihalins.

Les cours d'eau armoricains offrent des zones de reproduction et de croissance proches de la mer. Cependant, cette partie aval du réseau est fortement affectée par l'emprise de petits ouvrages rapprochés. Leur accumulation limite rapidement la progression des poissons migrateurs à l'intérieur du bassin et leurs biefs annihilent une grande partie du po- tentiel d'habitat disponible pour la reproduction des espèces potamotoques. A l'amont, les cours d'eau du massif central sont pénalisés par leur position continentale. En revanche, ces derniers présentent un potentiel productif bien conservé, sur de longs parcours "sauvages » relativement épargnés par l'emprise des retenues de barrage.

En raison des distances à la mer et du nombre des obstacles à franchir, le potentiel d'accueil et de production des habitats naturels est exposé aux phénomènes cumulatifs qui peuvent rapidement compromettre l'accès des géniteurs jusqu'aux frayères, de même que l'accès des stades dévalants jusqu'aux milieux marins. Sur des circuits migratoires de cette dimension, les taux de blocage et les retards concédés au niveau de chaque obstacle doivent être réduits au minimum, en particulier sur les $500 \mathrm{~km}$ d'axe principal qui séparent le bec d'Allier de l'estuaire.

Les opérations de restauration de libre circulation ont débuté à la fin des années 70 sur les grands axes de migration qualifiés pour le saumon au plan réglementaire (Loire-Allier et Vienne-Creuse-Gartempe). Les premiers résultats ont été contrariés par l'apparition de nouveaux points d'impacts (seuil de centrales nucléaires et plans d'eau de loisirs, radier de ponts affouillés par surcreusement du lit mineur, réarmement de micro-centrales, dégradation de la qualité de l'estuaire).

La restauration des populations migratrices très affaiblies par les 
effets cumulés exige d'importants efforts de rétablissement, de transparence migratoire et d'intégrité des milieux naturels aquatiques.

Des signes tangibles de reconquête sont observés aujourd'hui, à la suite d'opérations importantes réalisées dans le cadre du Plan Loire Grandeur Nature :

- recolonisation de la Creuse par la Lamproie et retour des premiers saumons sur la Gartempe après l'effacement du barrage de Maisons Rouges,

- reconquête de l'Allier supérieur par le saumon après l'aménagement du pont-barrage de Vichy et l'effacement du barrage de SaintEtienne-du-Vigan.

La transparence migratoire de la Loire moyenne est également en voie de rétablissement, grâce à la construction de passes en enrochements à la dimension du fleuve sur les seuils de centrales nucléaires.

Si des résultats significatifs ont été atteints en terme d'aire de répartition, l'état des stocks amphihalins reste aléatoire et conditionné par les facteurs hydrologiques exceptionnels qui peuvent réduire l'impact des effets cumulés.

Pour atteindre les objectifs fixés à l'échelle du bassin, il est donc nécessaire de poursuivre les efforts de restauration en évitant toute altération supplémentaire du réseau migratoire et en respectant les mesures de protection des stocks les plus sensibles.

\section{RÉFÉRENCES BIBLIOGRAPHIQUES}

Anonyme, 2001. Mesure de la qualité des cours d'eau, Réseau National de Bassin 1997-1999. Rapport Agence de l'Eau Loire-Bretagne, Direction Régionale de l'Environnement bassin LoireBretagne : 214 pp.

Anonyme, 2002. Sauvegarde du grand Saumon de Loire - projet 00NAT/F/7252 - rapport d'avancement couvrant la période du 1/01/01 au 31/12/01. Rapport LOGRAMI / CSP DR6 : 55 pp. + annexes.

Bach J.M. et al., 2001. Suivi des populations de saumons sur le bassin de l'Allier en 2000. Rapport LOGRAMI / CSP DR6 : 45 pp + annexes.

Carmie H., 1997. Restauration du saumon sur le bassin de la Loire. Volet repeuplements. CSP DR6 : 8 pp. + annexes

Chapon P.M., 1999. Suivi écologique de l'effacement du barrage de MaisonsRouges, observations réalisées et résultats obtenus concernant le retour des poissons migrateurs, bilan à la fin $1999: 7 \mathrm{pp}+$ illustrations.

Larinier M., Porcher J.P., Travade F., Gosset C., 1994. Passes à poissons, expertise, conception des ouvrages de franchissement. CSP, collection mise au point, Paris : $336 \mathrm{pp}$.

Menesson-Boisneau C., Boisneau P., 1990. Migration, répartition, reproduction, caractéristiques biologiques et taxonomie des aloses (Alosa sp.) dans le bassin de la Loire. Thèse de doctorat, Univ. Paris XII Val de Marne et Rennes I / INRA Rennes : 143 pp + annexes.

Menesson-Boisneau C., Boisneau P., POSTIC A., 1999. Abondance de la grande alose (Alosa alosa L. 1758) dans le Loire : analyse des facteurs de variabilité de 1984 à 1998, étude des caractéristiques biologiques des géniteurs de 1994 à 1998, mise au point 
d'un modèle de recrutement : $47 \mathrm{pp}+$ annexes.

Minster A.M., Bomassi P., 1999. Repérage et évaluation des surfaces potentielles de développement de juvéniles de saumons atlantiques. Proposition d'un modèle de gestion des stocks sur les bassins de l'Allier et de l'Arroux. Rapport LOGRAMI / CSP DR6 : 44 pp + annexes.

Sauriau P.G., 1990. Etude des populations de mulets dans l'estuaire de la Loire, bilan des études 1989-1990. Rapport APEEL, Université de Nantes laboratoire de biologie marine : $21 \mathrm{pp}$.
Souchon Y., Andriamahefa H., Cohen P., Breil P., Pella H., Lamouroux N., Malavoi J.R., Wasson J.G., 2000. Régionalisation de l'habitat aquatique dans le bassin de la Loire. Synthèse, Agence de l'Eau Loire-Bretagne, CEMAGREF - Département Gestion des Milieux Aquatiques - Groupement de Lyon : $291 \mathrm{pp}$.

Steinbach P., 1997. Situation des poissons migrateurs du bassin de la Loire, Contrat retour aux sources, actualisation 1996. Rapport CSP DR4 : 110 pp + annexes. 